\title{
A large deletion in the CFTR gene in CBAVD
}

Feras M. Hantash, MS, $P h D^{1}$, Aubrey Milunsky, MD, DSc ${ }^{2}$, Zhenyuan Wang, PhD ${ }^{2}$, Ben Anderson, $B S^{1}$, Weimin Sun, $P h D^{1}$, Arturo Anguiano, $M D^{1}$, and Charles M. Strom, $M D, P h D^{1}$

\begin{abstract}
Purpose: Most cystic fibrosis mutation screening methods do not detect large exon deletions or duplications in the cystic fibrosis transmembrane regulator gene. We looked for such mutations in congenital bilateral absence of the vas deferens patients in whom routine screening assays had identified only one or no cystic fibrosis transmembrane regulator gene mutations. Methods: DNA samples from 48 men with congenital bilateral absence of the vas deferens were tested for exonic deletions and duplications in the cystic fibrosis transmembrane regulator gene using a laboratory-developed semiquantitative fluorescent PCR assay. Results: Semi-quantitative fluorescent PCR identified a large deletion in one (2\%) of the 48 patients. This patient, previously characterized as carrying only the IVS8-5T mutation, was found to have a deletion of exons 22-24 of the cystic fibrosis transmembrane regulator gene. In a second patient with the IVS8-5T mutation, we identified a one-base pair insertion in exon 17b that disrupted the reading frame. Conclusions: Analysis of the cystic fibrosis transmembrane regulator gene for exon deletions and duplications should be included for complete study of CBAVD patients, especially those considering assisted reproduction. Genet Med 2006:8(2):93-95.
\end{abstract}

Key Words: CBAVD, CFTR, deletion, atypical CF, SQF PCR

Congenital bilateral absence of the vas deferens (CBAVD) is an atypical presentation of cystic fibrosis (CF). ${ }^{1}$ Mutations in the cystic fibrosis transmembrane regulator (CFTR) gene are recognized in about $85 \%$ of CBAVD cases ${ }^{2,3}$ and are present in $15 \%$ of patients with obstructive azoospermia. ${ }^{4}$ Studies indicate that in CBAVD patients harboring compound heterozygous CFTR mutations, the mutations are of the mild/severe type, while mutations of a homozygous or compound heterozygous severe/severe class are not detected. ${ }^{2,5,6}$ This is opposite to the detection of two mutations (homozygous or compound heterozygous) that convey severe phenotype in classic CF. ${ }^{7,8}$ In some CBAVD patients only one CFTR mutation can be detected, and it could be of the kind that presents with a severe or a mild phenotype. ${ }^{3,5}$

Several studies have shown that panel testing of the most common CFTR mutations will not detect mutations in a significant proportion of CBAVD patients. ${ }^{5,9}$ Extensive screening for mutations in CFTR exons using denaturing gradient gel electrophoresis (DGGE) followed by DNA sequencing will increase the CFTR mutation detection frequency, however, CFTR mutations will still not be detected in a subset of CBAVD patients. ${ }^{3,4}$ This could be due to the presence of mutations within introns not analyzed by the testing method or to the contribution of another gene to CBAVD. The latter possibility

From the ${ }^{I}$ Department of Genetics, Quest Diagnostics Nichols Institute, San Juan Capistrano, CA; and ${ }^{2}$ Center for Human Genetics, Boston University School of Medicine, Boston, MA.

Feras M. Hantash, MS, PhD, Department of Molecular Genetics, Quest Diagnostics Nichols Institute, San Juan Capistrano, CA 92690.

Received for publication June 8, 2005.

Accepted for publication November 29, 2005.

DOI: 10.1097/01.gim.0000200945.54234.d7 may be supported by a family study in which two brothers both carry $\Delta$ F508 and share identical haplotypes. One brother has CBAVD, while the other fathered children (confirmed by paternity testing).$^{10}$

Large rearrangements might be another cause of failure to detect CFTR mutations in patients with CBAVD. Most CFTR genotyping methods will not detect large mutations in the form of exon deletions or duplications, and the presence of such mutations has previously been described. ${ }^{11}$ In this study, we used a newly developed test for deletions and duplications in the CFTR gene to investigate CBAVD patients with one or no CFTR mutations identified by standard screening methods.

\section{MATERIALS AND METHODS}

\section{DNA samples}

Forty-nine anonymized DNA samples from CBAVD patients were studied. Initially, limited mutation panel testing was performed on these samples and twenty-four samples were previously determined to harbor one mutation (Group 1) and 25 samples were from patients with no identified CFTR mutations (Group 2). Genotypes from Group 1 were as follows: $\Delta$ F508/wt $(\mathrm{N}=13), 5 \mathrm{~T} / \mathrm{wt}(\mathrm{N}=7), \Delta$ F508/5T $(\mathrm{N}=1)$, G551D/wt genotype $(\mathrm{N}=2)$, and $\mathrm{N} 1303 \mathrm{~K} / \mathrm{wt}(\mathrm{N}=1)$. All samples were consequently analyzed using the CF-OLA version 3.0 kit (Applera), which detects 32 CFTR mutations, and the results were identical to initial genotyping data (data not shown). One sample from Group 2 consistently failed to amplify, presumably because of degraded or poor-quality DNA, and was excluded from the analysis. 


\section{Semi-quantitative fluorescent PCR (SQF PCR)}

In this assay, a single multiplex SQF PCR reaction is used for the detection of single or multiple exon deletions and duplications. Primers and PCR conditions are described in detail elsewhere. ${ }^{12}$ Briefly, samples were analyzed using two SQF PCR reactions and samples were analyzed twice. In the first reaction, primers representing the CFTR promoter and all 27 exons were amplified in a single reaction. Forward primers were labeled with FAM or HEX fluorescent dyes for analysis on ABI 3100 capillary instrument (Applied Biosystems). In the second SQF PCR reaction, fluorescently-labeled primers representing the promoter and exons 1-4, in addition to fragments upstream of the CFTR promoter were included in a single PCR reaction. Included in each SQF PCR reaction are three fragments representing the three internal control fragments from three different genes on different chromosomes for comparison and signal normalization. The internal control fragments were from Hexosamindase (Tay-Sachs), Factor $\mathrm{V}$ and Factor II genes. Cycling conditions were established to be in the linear phase of PCR. Amplified fragments were separated on an automated ABI 3100 instrument (Applied Biosystems) and data were analyzed using GeneMapper ${ }^{\circledR}$ software (Applied Biosystems). This method identifies CFTR mutations by detecting an approximate $50 \%$ drop in signal from deleted exon(s), and an approximate $30-50 \%$ increase in the signal of the duplicated exon $(\mathrm{s})$.

\section{Multiplex ligation-dependent probe amplification (MLPA)}

MLPA (MRC-Holland, Amsterdam, Holland; catalog \# P091), an assay designed to detect exon deletions and duplications, ${ }^{13}$ was used to confirm mutations identified by SQF PCR. Only samples suspected of harboring rearrangements were analyzed for confirmation. MPLA was performed according to the manufacturer's instructions; fragment and data analyses were performed as described for SQF PCR.

\section{DNA sequencing}

DNA sequencing was performed essentially as described by Strom et al. (2003), ${ }^{14}$ except that DNA purification post-PCR was done using exonuclease-calf intestinal alkaline phosphatase digestion, and post Dye-Term reaction DNA was purified by ethanol precipitation and resuspended in Hi-Di formamide. Samples were resolved on an ABI 3730 automated DNA analyzer and data were analyzed using SeqScape software (both from Applied Biosystems).

\section{RESULTS}

Using the SQF PCR method, we detected a large CFTR exon deletion in one (2\%) of the 48 samples tested: one sample from Group 1 had a deletion of exons 22, 23, and 24 of the CFTR gene (Online only - Fig. 1A,B), as shown by the drop in the signal of representative fragments when compared to a sample with no deletions. The presence of this large deletion was confirmed by MLPA (Online only - Fig. 1C). This sample had previously been characterized as harboring the IVS8-5T/7T al- leles. No exon duplications were detected in other Group 1 patients.

Samples were also analyzed for possible deletions or duplications using a separate SQF PCR reaction that included fragments of the promoter, exons 1-4 and three fragments within $\sim 5,000$ bp upstream of the CFTR promoter and ATG start codon. Samples did not show any large promoter deletions or duplications within the regions analyzed (data not shown).

SQF-PCR also led to detection of a point mutation in another Group 1 sample. This specimen displayed a decrease of exon $17 \mathrm{~b}$ signal and concurrent presence of a second peak immediately adjacent to the lower exon $17 \mathrm{~b}$ fragment (Online only - Fig. 2). An insertion of one base in exon $17 \mathrm{~b}$ was suspected, as this would explain the presence of the two adjacent presumptive exon 17b-related fragments. DNA sequencing using forward- and reverse-strand primers confirmed the presence of a heterozygous, one-base-pair insertion of adenosine in exon 17b at position 3372 (Genbank ID \# NM000492), leading to disruption of the CFTR reading frame. The patient also carries the IVS8-5T/7T alleles.

A third sample, from Group 2, displayed a reproducible drop in exon 19 fragment signal. Because the degree of signal reduction was not as great as would be expected from an exon deletion (data not shown), we suspected a polymorphism in the forward or reverse primer binding site of the exon 19 fragment. DNA sequence analysis identified a $3617 \mathrm{G}>$ T heterozygous base change that led to a R1162L mutation. This base change, located 4 bases from the $3^{\prime}$-end of the exon 19 forward primer, was described by Fanen et al. as a polymorphism, ${ }^{15}$ and was detected in a patient with asthma ${ }^{16}$ and in a patient with chronic pancreatitis. ${ }^{17}$ The R1162 residue is conserved across many species, ${ }^{16}$ and analysis of the effect of R1162L mutation on CFTR protein using the PolyPhen algorithm (http://www. bork.embl-heidelberg.de/PolyPhen/) suggested that this variant is probably damaging. The possibility exists that this mutation is of low penetrance.

\section{DISCUSSION}

In this study, SQF PCR identified a large exonic CFTR deletion in one of 48 (2\%) patients with isolated CBAVD in whom standard screening techniques had detected only one or no mutations. The deletion of exons 22-24 in this patient is predicted to remove the second nucleotide-binding domain (NBD2) and is therefore is predicated to severely influence CFTR protein function. Although the patient also carries the IVS8-5T allele, the phase of these alleles could not be determined. However, we have detected in two independent patients with classic CF symptoms a deletion of exons 22-24 who also harbor the $\Delta$ F508 mutation and the IVS8-7T/9T (F.M.H. data not shown). Since the $\Delta$ F508 almost always occurs on the IVS8-9T chromosome, it can be concluded that in our CBAVD proband, similar to the two CF cases, the deletion of exon 22-24 occurs on the IVS8-7T chromosome.

Even though we did not identify exon duplications in the DNA from the cohort of CBAVD patients we analyzed, we 
recently detected a large tandem duplication of exons $6 \mathrm{~b}-10$ in a classic CF patient. ${ }^{18}$ Therefore the SQF PCR method can detect both exon deletions and duplications.

Recently, Niel et al. described the complete deletion of one copy of the CFTR gene and the presence of $\mathrm{R} 117 \mathrm{H}(-7 \mathrm{~T})$ on the other chromosome in a 37-year-old man with CBAVD. ${ }^{19}$ This situation is predicted to result in reduced CFTR channel function due to the $\mathrm{R} 117 \mathrm{H}$ allele, ${ }^{8}$ and indeed this patient did not have pancreatic insufficiency or lung disease. ${ }^{19} \mathrm{~A}$ second CBAVD patient in that study harbored $\triangle \mathrm{F} 508$ and a deletion of exons 17-17b; however, this individual displays classic CF symptoms.

The CFTR SQF PCR method described here may also detect mutations in the form of single base-pair insertions or deletions in the majority of the exons. The second case we describe involved a heterozygous insertion of one base pair in exon $17 \mathrm{~b}$. In this case, the insertion changed the reading frame and led to the introduction of a stop codon 74 codons downstream. This would remove part of the second transmembrane domain (TM2), as well as NBD2, of the CFTR protein and is thus predicted to be a severe mutation. This mutation occurred in a patient harboring the IVS8-5T allele. The phase of the alleles is unknown. The 3372insA mutation appears to be novel, as it is not listed in the CFTR mutation database (http://www.genet. sickkids.on.ca/cftr/).

Because most comprehensive studies of CFTR mutations in CBAVD and obstructive azoospermia to date did not scan for large DNA rearrangements, ${ }^{5,6,9,10}$ the prevalence of such mutations may be underestimated. Indeed, common CFTR mutation panels have been shown to be inadequate in cases of obstructive azoospermia. ${ }^{9}$ Our results also point toward the importance of testing for large exonic deletions and duplications in cases of CBAVD in which no (or only one) mutation is identified by standard techniques. In our study, the exon 22-24 deletion would not have been detected by common screening approaches, including DNA sequencing, DGGE, and SSCP. If the proband's partner carries a CF mutation, any offspring conceived via assisted reproductive technology could receive two CFTR mutations.

In screening CBAVD patient for mutations in the CFTR gene, the decision to start with the college panel is an obvious one as it detects the most frequent mutations. Testing for the IVS8-5T can be done concurrent to panel testing or following that. What follows if further testing is needed is a decision of the doctor and the patient. Should a more comprehensive, time-consuming, and more expensive DNA sequencing test be requested, since this method can detect all point mutations and few base pair insertions and deletions, or should they choose to order, for example, the SQF PCR method, which gives faster results, is less costly, and, in our method, can detect large exon deletions or duplications and in most exons, single or few base pair insertions deletions, as described above. One can also decide to order dHPLC rather than apply full sequencing, but dHPLC will not detect large exon deletions or duplications, and a negative result on dHPLC does not rule out the presence of point mutations not detected under the conditions of the instrument, and DNA sequencing might still be required. If one were to devise a step-wise strategy, the only common elements of the strategy is to test on the ACMG/ACOG panels or expanded panels. What follows next is the sole decision of the doctor and the patient.

In summary, using SQF PCR we detected a large CFTR deletion not identified by standard screening in a patient with CBAVD. The results presented here and elsewhere ${ }^{11,19}$ highlight the importance of including screening for CFTR exon deletions and insertions in cases of CBAVD, especially for couples considering assisted reproductive technology.

\section{ACKNOWLEDGMENTS}

We would like to thank Jeff Radcliff for editorial assistance, and the reviewers for their helpful comments and suggestions.

\section{References}

1. Anguiano A, Oates RD, Amos JA, Dean M, et al. Congenital bilateral absence of the vas deferens. A primarily genital form of cystic fibrosis. JAMA 1992;267:1794-1797.

2. Chillon M, Casals T, Mercier B, Bassas L, et al. Mutations in the cystic fibrosis gene in patients with congenital absence of the vas deferens. N Engl J Med 1995;332:1475-1480.

3. Casals T, Bassas L, Egozcue S, Ramos MD, et al. Heterogeneity for mutations in the CFTR gene and clinical correlations in patients with congenital absence of the vas deferens. Hum Reprod 2000;15:1476-1483.

4. Kanavakis E, Tzetis M, Antoniadi T, Pistofidis G, et al. Cystic fibrosis mutation screening in CBAVD patients and men with obstructive azoospermia or severe oligozoospermia. Mol Hum Reprod 1998;4:333-337.

5. Wang Z, Milunsky J, Yamin M, Maher T, et al. Analysis by mass spectrometry of 100 cystic fibrosis gene mutations in 92 patients with congenital bilateral absence of the vas deferens. Hum Reprod 2002;17:2066-2072.

6. Claustres M, Guittard C, Bozon D, Chevalier F, et al. Spectrum of CFTR mutations in cystic fibrosis and in congenital absence of the vas deferens in France. Hum Mutat 2000;16:143-156.

7. Claustres M. Molecular pathology of the CFTR locus in male infertility. Reprod Biomed Online 2005;10:14-41.

8. Cutting GR. Cystic Fibrosis. In: DL Rmoin, JM Connor, RE Pyeritz, BR Korf, eds. Principles and Practice of Medical Genetics. Vol. 2 (ed 4). London: Churchill Livingstone; 2002:1561-1606

9. Mak V, Zielenski J, Tsui LC, Durie P, et al. Proportion of cystic fibrosis gene mutations not detected by routine testing in men with obstructive azoospermia. JAMA 1999;281:2217-2224.

10. Mercier B, Verlingue C, Lissens W, Silber SJ, et al. Is congenital bilateral absence of vas deferens a primary form of cystic fibrosis? Analyses of the CFTR gene in 67 patients. Am J Hum Genet 1995;56:272-277.

11. Audrezet MP, Chen JM, Raguenes O, Chuzhanova N, et al. Genomic rearrangements in the CFTR gene: extensive allelic heterogeneity and diverse mutational mechanisms. Hum Mutat 2004;23:343-357.

12. Hantash FM, Redman JB, Starn K, Anderson B, et al. Novel and recurrent rearrangements in the CFTR gene: clinical and laboratory implications for cystic fibrosis screening. Hum Genet. in press

13. Schouten JP, McElgunn CJ, Waaijer R, Zwijnenburg D, et al. Relative quantification of 40 nucleic acid sequences by multiplex ligation-dependent probe amplification. Nucleic Acids Res 2002;30:e57.

14. Strom CM, Huang D, Chen C, Buller A, et al. Extensive sequencing of the cystic fibrosis transmembrane regulator gene: assay validation and unexpected benefits of developing a comprehensive test. Genet Med 2003;5:9-14.

15. Fanen P, Ghanem N, Vidaud M, Besmond C, et al. Molecular characterization of cystic fibrosis: 16 novel mutations identified by analysis of the whole cystic fibrosis conductance transmembrane regulator (CFTR) coding regions and splice site junctions. Genomics 1992;13:770-776.

16. Lazaro C, de Cid R, Sunyer J, Soriano J, et al. Missense mutations in the cystic fibrosis gene in adult patients with asthma. Hum Mutat. 1999;14:510-519.

17. Casals T, Aparisi L, Martinez-Costa C, Gimenez J, et al. Different CFTR mutational spectrum in alcoholic and idiopathic chronic pancreatitis? Pancreas 2004;28:374-379.

18. Hantash FM, Redman JB, Sun W, Strom CM. Charecterization of a novel large duplication in the CFTR gene. Ped Pulmonol. Abs Ann Mtg NACFC. Baltimore, MD. 2005;S28. Abs. 180:251.

19. Niel F, Martin J, Dastot-Le Moal F, Costes B, et al. Rapid detection of CFTR gene rearrangements impacts on genetic counselling in cystic fibrosis. J Med Genet 2004; 41:e118. 